\title{
Altered long non-coding RNA expression profile in patients with IgA-negative mesangial proliferative glomerulonephritis
}

\author{
WEIGUO SUI ${ }^{1}$, HUAN LI $^{1,2}$, MINGLIN OU $^{1}$, DONG'E TANG $^{1,2}$ and YONG DAI ${ }^{3}$ \\ ${ }^{1}$ Nephrology Department, 181 Hospital, Guangxi Key Laboratory for Metabolic Diseases Research, Guilin 541002, \\ Guangxi; ${ }^{2}$ College of Life Science, Guangxi Normal University, Guilin 541004, Guangxi; ${ }^{3}$ Clinical \\ Medical Research Center, The Second Clinical Medical College of Jinan University \\ (Shenzhen People's Hospital), Shenzhen 518020, Guangdong, P.R. China
}

Received January 11, 2012; Accepted March 13, 2012

DOI: $10.3892 / \mathrm{ijmm} .2012 .975$

\begin{abstract}
Mesangial proliferative glomerulonephritis (MsPGN) is one of the most common immune-mediated renal diseases. The mesangium is expanded and hypercellular, immunoglobulin deposits can be found in the mesangium, but the mechanism underlying its cause remains largely unclear. There is a large amount of evidence suggesting that long >200 nucleotide) non-coding RNAs (lncRNA) have important regulatory functions in the epigenetic control of gene expression. Multiple lines of evidence increasingly link mutations and dysregulations of lncRNAs to a diverse number of human diseases. Through microarray expression analysis, tests show that thousands of lncRNAs and protein-coding genes are significantly differentially expressed in IgA-negative MsPGN. Some lncRNAs and their neighboring protein-coding genes are closely related and are cooperatively expressed. This may be part of a potential regulatory mechanism. The malfunction of regulation in the network of lncRNAs may be a possible mechanism for the development of IgA-negative MsPGN. Our observations suggest that some lncRNAs are closely related to IgA-negative MsPGN and may be playing an important role in this disease.
\end{abstract}

\section{Introduction}

Mesangial proliferative glomerulonephritis (MsPGN) is one of the most common immune-mediated renal diseases. Morphologically, MsPGN is characterized by diffuse mesangial cell proliferation and some increase in the mesangial matrix (1). These features are both detectable via immunohistochemical staining. IgA, IgM, IgG and complement component C3 depo-

Correspondence to: Dr Yong Dai, Clinical Medical Research Center, The Second Clinical Medical College of Jinan University (Shenzhen People's Hospital), Shenzhen 518020, Guangdong, P.R. China

E-mail: daiyong22@yahoo.com.cn

Key words: long non-coding RNA, mesangial proliferative glomerulonephritis, epigenetic regulation, transcription, renal sition can be observed in the glomerular mesangial area (2). Patients may present with varying amounts of hematuria and/ or proteinuria (2).

IgA nephropathy is the most common form of primary glomerulonephritis worldwide. We identified IgA-unassociated MsPGN when IgA deposition in the glomerular mesangial area is not observed. IgA-unassociated MsPGN is very common in China, but the mechanism underlying this disease remains largely unclear.

There is increasing evidence that long (>200 nucleotide) non-coding RNAs (lncRNAs) have important regulatory functions in mammalian biology (3). Multiple lines of evidence increasingly link mutations and dysregulations of lncRNAs to a diverse number of human diseases (4). Xist lncRNA is highly and specifically expressed by the inactive X-chromosome that ensures equal expression in both males and females (5). HOTAIR lncRNA binds to polycomb proteins to methylate histones at multiple HoxD loci. HOTAIR is also involved in the aberrant regulation of gene expression in cancer (6). SPRY4-IT1 lncRNA RNAi knockdown results in defects in cell growth, cell differentiation, and higher rates of apoptosis in melanoma cell lines (7). We were interested in the role of the dysregulations of IncRNAs in IgA-unassociated MsPGN.

This study describes an altered lncRNA expression profile in patients with IgA-unassociated MsPGN. We identified some dysregulations of lncRNAs associated with IgA-unassociated MsPGN. These dysregulations of lncRNAs may regulate neighboring protein-coding genes that are closely related to MsPGN. This research provides an important foundation for future research on MsPGN.

\section{Subjects and methods}

Patients and healthy controls. Renal biopsies were obtained from IgA-unassociated MsPGN in patients (ages, 18-29) at the 181 Hospital in Gulin, China, between March and September 2010. The diagnosis of IgA-unassociated MsPGN was confirmed through pathological diagnosis and the presence of other clinical evidence. All of the patients in this study presenting to the Nephrology Department of the 181 Hospital had renal biopsies positive for IgA-unassociated MsPGN. All patients in this study were diagnosed without any other complications. The patients 
had never been treated with immunosuppressive drugs. Four of the biopsies performed were classified as lupus nephritis based on the clinical definitions from 2003 (8). Renal biopsies were performed according to clinical indications using ultrasound observation and a biopsy instrument. One sample from the control group was of a section of renal cortex obtained from a renal resection operation. This tissue suffered serious cuts, but appeared normal upon screening with a light microscope. Written informed consent was obtained from all subjects or their guardians. Use of biopsy material for further studies beyond routine diagnosis was approved by the local ethics committee. This study was performed according to the guidelines of the 181 Hospital. These guidelines abide by the Helsinki Declaration on ethical principles for medical research involving human subjects.

Histological analysis. Biopsy material was immediately fixed in $10 \%$ phosphate-buffered paraformaldehyde and stored at $4^{\circ} \mathrm{C}$. After fixation, biopsies were dehydrated through an ascending ethanol series and were embedded in Epon 812. Serial, semi-thin sections $(0.5 \mu \mathrm{m}$ thick $)$ were cut on a Reichert Ultracut E microtome. Resin was removed by treating the sections with sodium methoxide prior to rehydration and immunostaining as previously described.

Preparation of renal tissue samples. Aspiration-needle biopsies from lupus nephritis patients and renal cortex pieces $\left(<0.3 \times 0.3 \times 0.3 \mathrm{~mm}^{3}\right)$ obtained post-nephrectomy were immediately washed with $0.9 \% \mathrm{NaCl}$ (RNase-free) and dipped in RNase inhibitor (Epicentre, USA) according to the manufacturer's instructions. The samples were stored at $4^{\circ} \mathrm{C}$ overnight. Depressors were removed from the biopsies and stored at $-80^{\circ} \mathrm{C}$ until needed for further testing.

Specimen collection processing. Samples were lysed in cell disruption solution and centrifuged at low speed (1,500 rpm). The upper aqueous layer was then removed and processed immediately in an RNA isolation procedure.

RNA isolation and target labeling. Total-RNAs were obtained using TRIzol (Invitrogen, USA) and the RNeasy kit (Qiagen, Germany) according to the manufacturer's instructions. This extraction included a DNase digestion step. After passing RNA measurement quality control on the NanoDrop ND-1000 and denaturing gel electrophoresis, the RNA was used to synthesize double-stranded cDNA using the SuperScript Double-Stranded cDNA Synthesis kit (Invitrogen). Double-stranded cDNA was labeled and hybridized to a $12 \times 135 \mathrm{~K}$ lncRNA expression microarray using the NimbleGen Hybridization system.

Microarray expression analysis. The microarray used is designed for the global profiling of long transcripts, including lncRNAs and protein-coding mRNAs. Each transcript is represented by $1-5$ unique probes used to improve statistical confidence in the result. Probes for reference genes and negative probes are printed multiple times to ensure hybridization quality. Human lncRNAs $(n=18,534)$ were collected from authoritative data sources including NCBI RefSeq, UCSC, RNAdb, from the literature and UCRs. Sequences from these data sources are selected carefully using special strategies. Highly similar sequences and ncRNAs shorter than 200 bp are excluded. Protein coding genes $(n=18,847)$ from NCBI RefSeq are also present on this array. This enables the detection of mRNAs and lncRNAs in a single experiment.

Raw data were extracted as pair files by NimbleScan software (version 2.5). NimbleScan software's implementation of RMA offers quantile normalization and background correction of data. Probe level normalization ('_norm_RMA.pair) files and gene summary ('_RMA.calls) files were produced. The gene summary files were imported into Agilent GeneSpring Software (version 11.0) for further analysis. Differentially expressed genes were identified through fold-change screening.

Statistical analysis. Signal intensities for each spot were analyzed and calculated by the Axon GenePix 4000B Microarray Scanner (Axon, USA), the NimbleScan software (version 2.5, Roche NimbleGen, Inc., USA) and the Agilent GeneSpring Software (version 11.0, Agilent, USA). Signal intensities for each spot were scanned and calculated by subtracting local background (based on the median intensity of the area surrounding each spot) from the total intensities. An average value of the 1-5 spot replicates of each lncRNA was generated after data transformation (to convert any negative value to 0.01 ). Normalization was performed using a per chip 50 th percentile method that normalizes each chip to its median. This normalization allows the user to compare across chips. To highlight lncRNAs that characterize each group, a per gene on median normalization was performed. This normalizes the expression of every lncRNA by its median across samples.

Pathway analysis and gene ontology $(G O)$ term analysis by differentially expressed mRNAs. Pathway analysis is based on the latest Kyoto Encyclopedia of Genes and Genomes (KEGG) database. This analysis allows users to determine the biological pathways involved with their differentially expressed mRNAs.

GO analysis is a functional analysis that associates differentially expressed mRNAs with GO categories. The GO categories are derived from Gene Ontology (www.geneontology.org), which comprises three structured networks of defined terms used to describe gene product attributes.

The P-value denotes the significance of the pathway and the GO term enrichment in the differentially expressed mRNA list. The lower the P-value, the more significant the pathway and the GO term (P-value $\leq 0.05$ is recommended).

Quantitative real-time RT-PCR. Total-RNA extracted from various samples was 2 -fold serially diluted in nuclease-free water. The diluted total-RNA was used as a template for realtime RT-PCR. Data were collected in duplicate for each sample. Master mix without total-RNA was prepared for all reactions with $24 \mu \mathrm{l}$ being aliquoted into each reaction tube. The diluted total-RNA of $1 \mu \mathrm{l}$ was added to each reaction. Reactions were conducted using the Rotor-Gene 3000 Real-time PCR system (Corbett Research) with the following reaction profile: cDNA synthesis for $60 \mathrm{~min}$ at $37^{\circ} \mathrm{C}$; pre-denaturation for $5 \mathrm{~min}$ at $95^{\circ} \mathrm{C}$; and PCR amplification for 40 cycles with $10 \mathrm{sec}$ at $95^{\circ} \mathrm{C}$, $15 \mathrm{sec}$ at $58^{\circ} \mathrm{C}$, and $20 \mathrm{sec}$ at $72^{\circ} \mathrm{C}$. The PCR was followed by a melting curve analysis to determine the reaction specificity. Agarose gel electrophoresis was performed to confirm the size of the PCR product. The mean $\mathrm{Ct}$ value was determined after the reaction to test the linearity of the GAPDH expression level. 

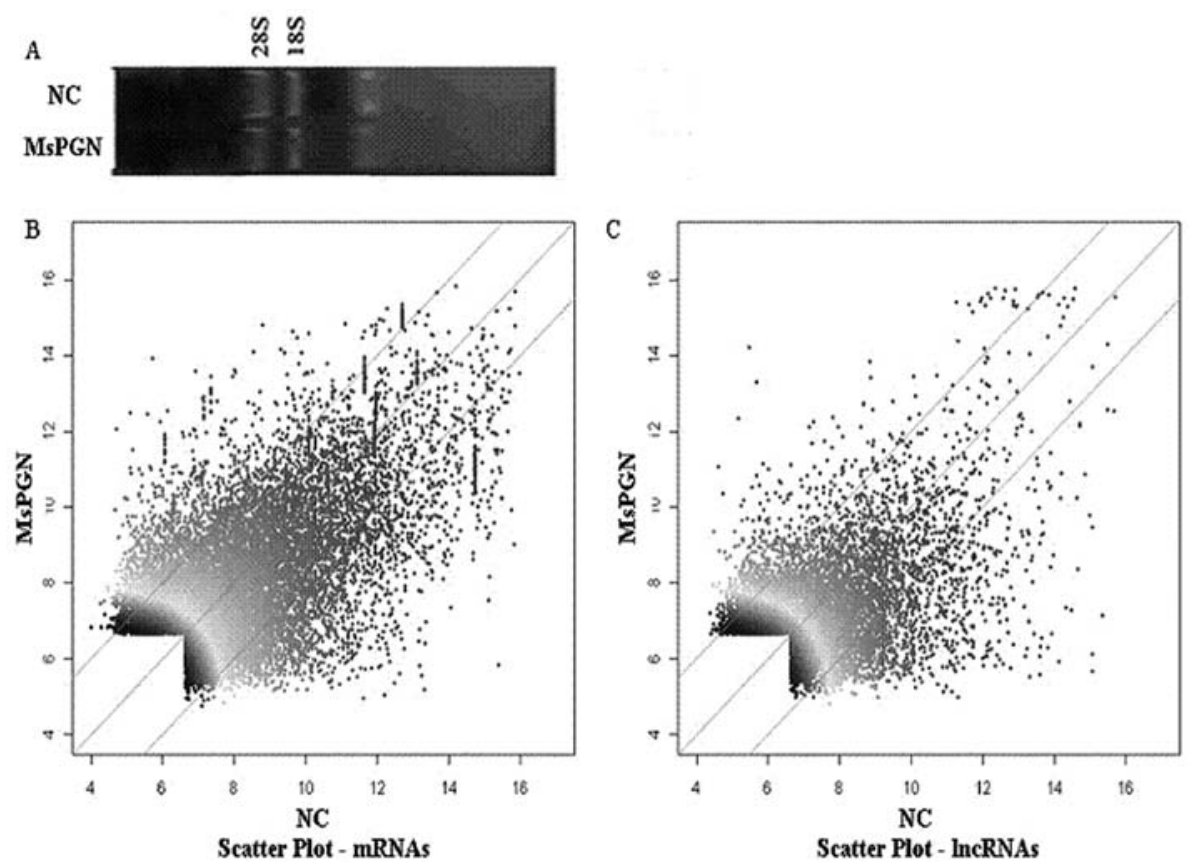

Figure 1. (A) Gel electrophoresis check of pooled total-RNA from MsPGN and NC samples. The scatterplots show (B) protein coding mRNA variation and (C) lncRNA variation between the MsPGN and NC chips.

The following primers were used to quantify the CREB binding protein (CREBBP) and AK130579 (CREBBPncRNA) mRNA levels: CREBBP, 5'-TACCTGTGCTGGAA ATGAAGAC-3' (forward) and 5'-GCTGTGCCGTTACTGC TACTC-3' (reverse); AK130579, 5'-GCTGCTCATCTTAGTG CTTCG-3' (forward) and 5'-CCACCACTTGCTAGGGATT TAC-3' (reverse). GAPDH RNA was quantified as a control to normalize differences in total RNA levels using the following primers: 5'-GGGAAACTGTGGCGTGAT-3' (forward) and 5'-GAGTGGGTGTCGCTGTTGA-3' (reverse).

IncRNA relative to adjacent protein-coding gene analysis. lncRNA originated from complex transcriptional loci. We analyzed IncRNA relative to adjacent protein-coding genes and found that there were 3 groups. i) The cis-antisense lncRNAs were from the same genomic locus as their target transcript but from the opposite DNA strand. cis-antisense lncRNAs form perfect or piece pairs with their target. ii) Intronic lncRNAs were mapped within the intron of a protein-coding gene. iii) Promoterassociated lncRNAs were defined where the lncRNA transcript crossed the protein-coding gene transcription start site.

\section{Results}

Differential expression of thousands of IncRNAs in IgA-unassociated MsPGN. The quantity and quality of the RNA samples in IgA-unassociated MsPGN and normal control (NC) groups were checked by gel electrophoresis (Fig. 1A). The gel electrophoresis results confirmed the good quality of the total-RNA isolated. Microarray expression analysis showed the protein coding mRNA and lncRNA variation between the MsPGN chips and the NC chips (Fig. 1B and C).

In this study, we performed fold-change filtering between the patients and the control samples from the experiment to identify differentially expressed lncRNAs in IgA-unassociated
MsPGN. The threshold we used to screen up or downregulated genes was fold-change $\geq 4.0$ for mRNA transcripts and fold-change $\geq 3.0$ for IncRNAs. After normalization and fold-change filtering, we found that $16.42 \%(3,095 / 18,847)$ of protein-coding transcripts in IgA-unassociated MsPGN biopsy samples were significantly differentially expressed (fold-change $\geq 4.0)$ and $15.37 \%(2,848 / 18,534)$ of lncRNA transcripts were significantly differentially expressed.

IncRNA relative to adjacent protein-coding genes analysis. IncRNAs do not code for proteins and fulfill functional roles that include high order chromosomal dynamics, telomere biology and sub-cellular structural organization (9). Some evidence suggests that lncRNAs can regulate gene expression through epigenetic regulation of chromatin modification, transcription and post-transcriptional processing $(10,11)$. In this study, we focus on the genomic context of IncRNAs. We aimed to analyze complex transcriptional loci that include lncRNAs and their associated protein-coding genes. This study may help reveal lncRNAs function in IgA-unassociated MsPGN and serve as a useful tool as we explore the mechanisms underlying IgA-unassociated MsPGN.

cis-antisense lncRNAs. There are a large number of complex loci in the mammalian genome. The cis-regulatory impact on cis-antisense genes is of functional importance (12). Current evidence has shown a variety of regulatory roles for cisantisense lncRNAs like reprogramming of chromatin, RNA interference (RNAi), alternative splicing, genomic imprinting, and $\mathrm{X}$-chromosome inactivation (13-16). In data from this study, we found that approximately $24 \%$ of expressed lncRNAs were cis-antisense lncRNAs.

We found an IncRNA (AF1180924, FGG-ncRNA) that was identified as being antisense to the fibrinogen $\gamma$ chain, FGG (Fig. 2A). FGG is a member of the complement and coagulation 


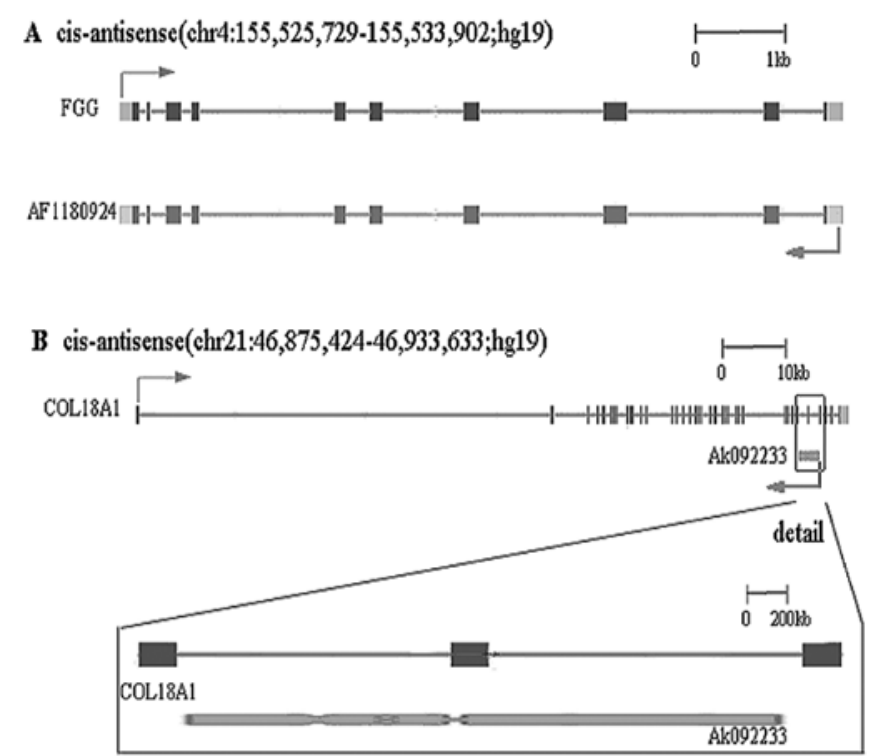

Figure 2. IncRNA lies opposite to the associated protein-coding gene. (A) FGG and FGG-ncRNA (AF1180924) genomic locus. (B) COL18A1 and COL18A1-ncRNA (AK092233) genomic locus.

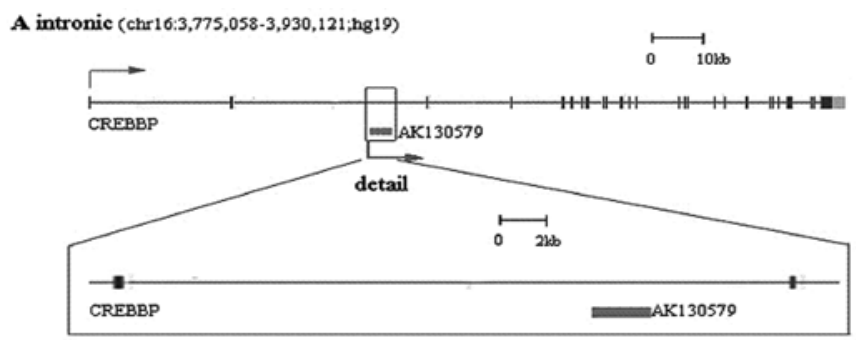

B intronic (chr1:65,886,318-66,102,820; $\mathrm{hg} 19)$

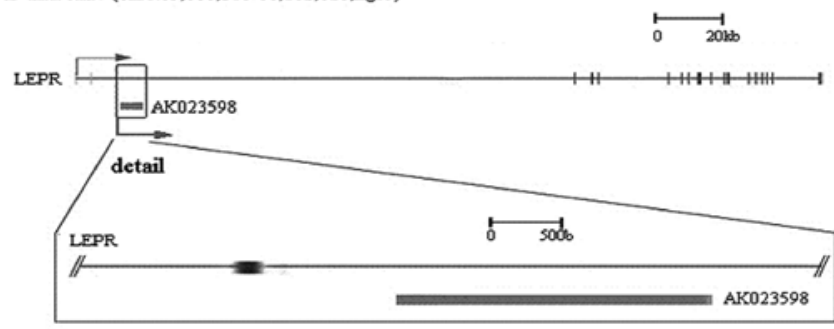

Figure 3. IncRNA within the introns of associated protein-coding gene. (A) CREBBP and CREBBP-ncRNA (AK130579) genomic locus. (B) LEPR and LEPR-ncRNA (AK023598) genomic locus.

cascade pathway that regulates cell adhesion and spreading, displays vasoconstrictor and chemotactic activities, and acts as a mitogen for several cell types. FGG binds factors, such as fibroblast growth factor-2 (FGF-2) and vascular endothelial growth factor (VEGF), cytokines, such as interleukin (IL)-1, and it is a high affinity receptor on stimulated monocytes and neutrophils. These binding interactions can also contribute to pathophysiological processes including inflammation and thrombosis $(17,18)$. The antisense ncRNA, which we named FGG-ncRNA, overlaps with the FGG in IgA-unassociated MsPGN (Fig. 2A). We found that increased expression of FGG was associated with proliferation of mesangial cells, and promoter-associated(chr2:37,870,743-37,899,326;hg19)

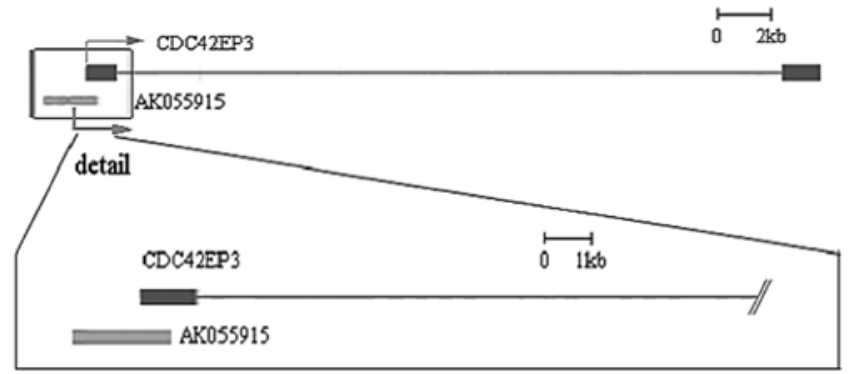

Figure 4. IncRNAs across the promoter of associated protein-coding genes, CDC42EP3 and CDC42EP3-ncRNA (AK055915) genomic locus.

the increased expression of FGG-ncRNA. This observation suggests that FGG and FGG-ncRNA are both associated with this disease, but might be independently regulated.

We also identified another antisense ncRNA (AK092233, COL18A1-ncRNA) that lies opposite of the collagen type XVIII $\alpha 1$ (COL18A1) gene in a highly complex locus (Fig. 2B). COL18A1 encodes the $\alpha$ chain of collagen type XVIII. Proteolytic cleavage within type XVIII collagen C-terminal domain releases the fragment endostatin which has antiangiogenic properties (19). Collagen XVIII is a heparan sulphate proteoglycan that is expressed ubiquitously in different basement membranes throughout the body. Increasing evidence shows that COL18A1 is important in other organs during non-physiological situations like atherosclerosis, glomerulonephritis or other types of tissue damage (20). Anti-GBM disease upregulates collagen XVIII/endostatin expression within the GBM and Bowman's capsule. Collagen XVIII/ endostatin preserves the integrity of the extracellular matrix and capillaries in the kidney, protecting against progressive glomerulonephritis (19). In this study, we found that COL18A1 gene expression was highly upregulated, but the expression of its antisense ncRNA, which we named COL18A1-ncRNA, was drastically downregulated. AK092233 may negatively regulate the COL18A1 gene.

Intronic lncRNAs. At complex loci, we observed a large number of lncRNAs within the introns of protein-coding genes. LncRNAs from introns have diverse regulatory functions, including being the precursors of shorter RNAs, proteincoding RNA stabilization, the control of gene expression, and the regulation of alternative splicing in protein-coding RNA (21-24). For example, we identified an ncRNA (AK130579, CREBBP-ncRNA) located within the introns of CREBBP (Fig. 3A). CREBBP is a member of the adherens junction and the Wnt signaling pathway. It is ubiquitously expressed and is involved in the transcriptional co-activation of many different transcription factors. CREBBP is a bimodal regulator of Wnt signaling and control both proliferation and differentiation through their interactions with partner $\beta$-catenin $(25,26)$. In our study, we observed downregulation in CREBBP gene expression. Expression of its intronic ncRNA, which we named CREBBP-ncRNA, was upregulated. Quantitative real-time RT-PCR experimental results validated these observations. We inferred that AK130579 may negatively regulate the CREBBP gene. 
We also identified another intronic ncRNA (AK023598, LEPR-ncRNA) that was located within the introns of the leptin receptor gene (LEPR) (Fig. 3B). The LEPR-encoded protein belongs to the gp130 family of cytokine receptors. Leptin binding to the leptin receptor protein activates the associated Jak/STAT signaling pathway (27). Leptin triggers a paracrine interaction in which glomerular endothelial cells secrete TGF- $\beta$. This secretion causes a response in sensitized mesangial cells (28). LEPR-encoded proteins indirectly control leptin activity (27). In this study, we found that LEPR gene expression was downregulated, but the expression of its intronic ncRNA, which we named LEPR-ncRNA, was upregulated. From these data we inferred that AK023598 may negatively regulate the LEPR gene.

Promoter-associated IncRNAs. IncRNAs interact with the downstream promoter region of protein-coding genes, thus regulating mRNA expression (29). Such an example is a lncRNA (AK055915) across the transcription start site of CDC42 effector protein 3 (CDC42EP3), which we named CDC42EP3-ncRNA (Fig. 4). CDC42 is a member of the Rho GTPase family of intracellular molecular switches. It is responsible for regulating multiple signaling pathways and is involved in actomyosin organization and cell proliferation (30). Rho GTPase activity and expression are critical in the development and function of the kidney (31). The CDC42EP3-encoded protein can regulate the function of $\mathrm{CDC} 42$. Interestingly, we observed that CDC42EP3 and CDC42EP3-ncRNA are both upregulated in IgA-unassociated MsPGN, possibly through independently regulated mechanisms.

\section{Discussion}

Using high-throughput microarray technologies designed for genome-wide identification, a large number of non-coding RNAs (ncRNAs) were discovered. In the past, people paid close attention to DNA and the catalytic functions of proteins. Now, the significance of ncRNAs is gradually becoming recognized. In mammals, ncRNAs occupy the majority of human transcriptional units (32). Many classes of ncRNAs are now extensively studied and their regulatory role is broadly recognized. IncRNA is an important class of ncRNA. Similarly to well-known miRNAs and piRNAs, IncRNAs have important regulatory functions in mammalian biology. Recently, evidence has shown that IncRNA can modulate gene expression through epigenetic regulation. Many lncRNAs that have functional significance are conserved (33). Under the influence of different evolutionary constraints, lncRNAs that are not as well conserved do not necessarily lack important functions (34).

IgA-unassociated MsPGN is a multisystem autoimmune disease whose mechanism is incompletely understood. The common view is that the autoantibody binds to antigens in IgA-unassociated MsPGN, then, forming immune complexes. The immune complex is deposited in the mesangial area and mediates cell and tissue injury (2). IncRNAs have been shown to play important roles in many diseases $(4,35)$. SPRY4-IT1 (7), HOTAIR (14), and HULC (36) for example, are associated with protein-coding genes where they cooperatively regulate gene expression. In this study, we observed thousands of differentially expressed lncRNAs in the IgA-unassociated MsPGN. Hundreds of these lncRNAs were associated with protein-coding genes and were cooperatively expressed among the total IncRNAs. As analyzed above, these IncRNAs affect the expression of their associated protein-coding genes. The malfunction of regulation in the network of lncRNAs may be a possible mechanism for the provocation of IgA-unassociated MsPGN. Nevertheless, it is important to note that the findings described in this article are merely a starting point for the study of long ncRNAs in IgA-unassociated MsPGN. Exciting studies lie ahead in the functional characterization of the identified ncRNAs.

\section{Acknowledgements}

We would like to thank the patients and healthy volunteers who participated in this study. This study was supported by the Guangxi of China Natural Science Fundation (2011GXNSFB018105).

\section{References}

1. Schwartz MM: The pathology of lupus nephritis. Semin Nephrol 27: 22-34, 2007.

2. Weening JJ, D'Agati VD, Schwartz MM, et al: The classification of glomerulonephritis in systemic lupus erythematosus revisited. Kidney Int 65: 521-530, 2004.

3. Hung T and Chang HY: Long noncoding RNA in genome regulation: prospects and mechanisms. RNA Biol 7: 582-585, 2010.

4. Wapinski $\mathrm{O}$ and Chang HY: Long noncoding RNAs and human disease. Trends Cell Biol 21: 354-361, 2011.

5. Pontier DB and Gribnau J: Xist regulation and function explored. Hum Genet 130: 223-236, 2011.

6. He S, Liu S and Zhu H: The sequence, structure and evolutionary features of HOTAIR in mammals. BMC Evol Biol 11: 102, 2011.

7. Khaitan D, Dinger ME, Mazar J, Crawford J, Smith MA, Mattick JS and Perera RJ: The melanoma-upregulated long noncoding RNA SPRY4-IT1 modulates apoptosis and invasion. Cancer Res 71: 3852-3862, 2011.

8. Markowitz GS and D'Agati VD: Classification of lupus nephritis. Curr Opin Nephrol Hypertens 18: 220-225, 2009.

9. Amaral PP and Mattick JS: Noncoding RNA in development. Mamm Genome 19: 454-492, 2008.

10. Rinn JL, Kertesz M, Wang JK, et al: Functional demarcation of active and silent chromatin domains in human HOX loci by noncoding RNAs. Cell 129: 1311-1323, 2007.

11. Mercer TR, Dinger ME and Mattick JS: Long non-coding RNAs: insights into functions. Nat Rev Genet 10: 155-159, 2009.

12. Engström PG, Suzuki H, Ninomiya N, et al: Complex loci in human and mouse genomes. PLoS Genet 2: e47, 2006.

13. Zhang Y, Liu XS, Liu QR and Wei L: Genome-wide in silico identification and analysis of cis natural antisense transcripts (cisNATs) in ten species. Nucleic Acids Res 34: 3465-3475, 2006.

14. Gupta RA, Shah N, Wang KC, et al: Long non-coding RNA HOTAIR reprograms chromatin state to promote cancer metastasis. Nature 464: 1071-1076, 2010.

15. Ogawa Y, Sun BK and Lee JT: Intersection of the RNA interference and X-inactivation pathways. Science 320: 1336-1341, 2008.

16. Sheik Mohamed J, Gaughwin PM, Lim B, Robson P and Lipovich L: Conserved long noncoding RNAs transcriptionally regulated by Oct 4 and Nanog modulate pluripotency in mouse embryonic stem cells. RNA 16: 324-337, 2010.

17. Farrell DH: Pathophysiologic roles of the fibrinogen gamma chain. Curr Opin Hematol 11: 151-155, 2004.

18. Mosesson MW: Fibrinogen and fibrin structure and functions. J Thromb Haemost 3: 1894-1904, 2005.

19. Hamano Y, Okude T, Shirai R, et al: Lack of collagen XVIII/ endostatin exacerbates immune-mediated glomerulonephritis. J Am Soc Nephrol 21: 1445-1455, 2010.

20. Seppinen L and Pihlajaniemi T: The multiple functions of collagen XVIII in development and disease. Matrix Biol 30: 83-92, 2011.

21. Rodriguez A, Griffiths-Jones S, Ashurst JL and Bradley A: Identification of mammalian microRNA host genes and transcription units. Genome Res 14: 1902-1910, 2004. 
22. Li SC, Tang P and Lin WC: Intronic microRNA: discovery and biological implications. DNA Cell Biol 26: 195-207, 2007.

23. Singh R: RNA-protein interactions that regulate pre-mRNA splicing. Gene Expr 10: 79-92, 2002.

24. Nakaya HI, Amaral PP, Louro R, et al: Genome mapping and expression analyses of human intronic noncoding RNAs reveal tissue-specific patterns and enrichment in genes related to regulation of transcription. Genome Biol 8: R43, 2007.

25. Li J, Sutter C, Parker DS, Blauwkamp T, Fang M and Cadigan KM: $\mathrm{CBP} / \mathrm{p} 300$ are bimodal regulators of Wnt signaling. EMBO J 26 : 2284-2294, 2007.

26. Teo JL and Kahn M: The Wnt signaling pathway in cellular proliferation and differentiation: A tale of two coactivators. Adv Drug Deliv Rev 62: 1149-1155, 2010.

27. Villanueva EC and Myers MG Jr: Leptin receptor signaling and the regulation of mammalian physiology. Int J Obes 32 (Suppl 7) S8-S12, 2008.

28. Wolf G, Chen S, Han DC and Ziyadeh FN: Leptin and renal disease. Am J Kidney Dis 39: 1-11, 2002.

29. Abarrategui I and Krangel MS: Noncoding transcription controls downstream promoters to regulate T-cell receptor alpha recombination. EMBO J 26: 4380-4390, 2007.
30. Melendez J, Grogg M and Zheng Y: Signaling role of Cdc42 in regulating mammalian physiology. J Biol Chem 286: 2375-2381, 2011.

31. Bielek H, Anselmo A and Dermardirossian C: Morphological and proliferative abnormalities in renal mesangial cells lacking RhoGDI. Cell Signal 21: 1974-1983, 2009.

32. Birney E, Stamatoyannopoulos JA, Dutta A, et al: Identification and analysis of functional elements in $1 \%$ of the human genome by the ENCODE pilot project. Nature 447: 799-816, 2007.

33. Guttman M, Amit I, Garber M, et al: Chromatin signature reveals over a thousand highly conserved large non-coding RNAs in mammals. Nature 458: 223-227, 2009.

34. Pang KC, Frith MC and Mattick JS: Rapid evolution of noncoding RNAs: lack of conservation does not mean lack of function. Trends Genet 22: 1-5, 2006.

35. Gibb EA, Brown CJ and Lam WL: The functional role of long non-coding RNA in human carcinomas. Mol Cancer 10: 38, 2011.

36. Wang J, Liu $\mathrm{X}, \mathrm{Wu} \mathrm{H}$, et al: CREB upregulates long non-coding RNA, HULC expression through interaction with microRNA372 in liver cancer. Nucleic Acids Res 38: 5366-5383, 2010. 